\title{
COMENTARIOS SOBRE EL ARTÍCULO: "AGENTES RELACIONADOS A INFECCIÓN DE SITIO OPERATORIO EN ADULTOS MAYORES POS OPERADOS EN EL CENTRO MÉDICO NAVAL, 2013 - 2017"
}

\author{
COMMENTS ON THE ARTICLE: "AGENTS RELATED TO INFECTION OF THE OPERATING SITE IN POST- \\ OPERATED MAJOR ADULTS IN THE NAVAL MEDICAL CENTER, 2013 - 2017"
}

Jordy Pulido-Flores' ${ }^{1}$, Andre Ore-Arce', Jacqueline Reyes-Gamonal'

\begin{abstract}
Sr. Editor
Tras leer con interés el artículo publicado por Hidalgo et al', referente a los agentes relacionados a infección de sitio operatorio (ISO) en adultos mayores post operados en el centro médico naval entre los años 2013 y 2017; encontramos la consideración de ciertos determinantes de relevancia relacionados al aspecto clínico y quirúrgico. Estos determinantes

son útiles, ya que pueden ser tomados como referencia en trabajos de investigación a futuro. Sin embargo, nos gustaría enfatizar la necesidad de incorporar algunas otras variables de relevancia como posibles determinantes a controlar, como lo son las relacionadas al estado nutricional, en específico los niveles de hemoglobina y albumina pre operatoria, ya que en nuestro país este tipo de patologías son habituales.
\end{abstract}

La ISO es una de las complicaciones más mortales y costosas en diferentes países 2, llegando hasta a incrementar la mortalidad de 2 a 11 veces 3 y, aumentando el costo hasta 14,2 millones de dólares en cirugías ${ }^{4}$. En el Perú, de 15000 infecciones intrahospitalarias, el 30,9\% son de sitio operatorio ${ }^{5}$. Es importante conocer los detalles de este tema debido a que constituye un problema social y económico altamente reversible.

El estado de nutrición del adulto mayor es vital para mantener una respuesta inmune adecuada ${ }^{6}$, una alteración habitual es la anemia, un frecuente problema de salud pública en países subdesarrollados como el Perú ${ }^{7}$ y constituye un factor de riesgo significativo que puede aumentar la fragilidad de una persona ${ }^{8,9}$ y así incrementar las probabilidades del desarrollo de una $\mathrm{ISO}^{10}$. El nivel de hemoglobina pre quirúrgica bajo los valores considerados normales tiene una estrecha asociación a las complicaciones quirúrgicas, como se evidencia en el estudio de Atalaya Marín donde del $49.5 \%$ de pacientes anémicos, el $67.9 \%$ presenta complicaciones postquirúrgicas en la que se destaca la $\mathrm{ISO}^{11}$.

Por otro lado, los valores de albúmina son de gran utilidad para poder determinar el estado nutricional de los pacientes en general ${ }^{12}$ ya que está a disposición en los centros hospitalarios. Los pobres niveles de albúmina han sido relacionados a pacientes con alteraciones de cicatrización debido a la alteración en la síntesis de colágeno y así impide la formación del granuloma en las heridas quirúrgicas, predisponiendo a la contaminación bacteriana en el ambiente hospitalario ${ }^{13}$. Si bien se conoce que la albúmina se reduce en una respuesta de fase aguda, no se pueden establecer conclusiones sobre si los niveles preoperatorios de prealbúmina podrían predecir el desarrollo de una infección ${ }^{14}$. A pesar de ello, diferentes estudios han descrito que el déficit de albúmina incrementa el riesgo de ISO hasta 12,6 veces más ${ }^{13}$. Además, queda constatado que los pacientes con valores de albúmina sérica postoperatorio $<3.5 \mathrm{~g} / \mathrm{dL}$ tienen un riesgo significativamente mayor de mortalidad por $\mathrm{ISO}^{14}$.

Independiente de que el estado peruano está lidiando con la malnutrición, es necesario intensificar esfuerzos para evitar este tipo de complicaciones, que perjudican social y económicamente. Creemos necesario enfatizar el estado nutricional de la persona como un factor de riesgo para desarrollar ISO en futuros trabajos a desarrollarse.

${ }^{1}$ Facultad de Medicina Humana, Universidad Ricardo Palma, Lima-Perú.

Citar como: Jordy Pulido-Flores, Andre Ore-Arce, Jacqueline Reyes-Gamonal. Comentarios sobre el artículo: "agentes relacionados a infección de sitio operatorio en adultos mayores pos operados en el Centro Médico Naval, 2013 - 2017". Rev. Fac. Med. Hum. Octubre 2019; 19(4):138-139. DOI 10.25176/RFMH.v19i4.2348 
Contribuciones de autoría: los autores participaron en la generación, recolección de información, redacción y versión final del artículo original.

Financiamiento: Autofinanciado.

\author{
Correspondencia: Jordy Pulido-Flores \\ Dirección: Av. Alfredo Benavides 5440, Santiago de Surco, Lima-Perú. \\ Teléfono:920238351 \\ Correo:jpulidoflores@gmail.com
}

Conflicto de interés: Los autores declaran no tener conflictos de interés en la publicación de este artículo.

Recibido: 28 de agosto del 2019

Aprobado: 10 de setiembre del 2019

\section{REFERENCIAS BIBLIOGRÁFICAS}

1. Lucía Fernanda Hidalgo-Vizarreta, Magdiel José Manuel GonzalesMenéndez, Cecilia Roxana Salinas-Salas. Agentes relacionados a infección de sitio operatorio en adultos mayores pos operados en el centro médico naval, 2013 - 2017. Rev. Fac. Med. Hum. Julio 2019; 19(3):43-47. Disponible en: 10.25176/RFMH.v19i3.2163

2. Berríos-Torres SI, Umscheid CA, Bratzler DW, Leas B, Stone EC, Kelz RR, et al. Centers for Disease Control and Prevention Guideline for the Prevention of Surgical Site Infection, 2017. JAMA Surg. 1 de agosto de 2017;152(8):784-91. Disponible en: 10.1001/jamasurg.2017.0904

3. Ban KA, Minei JP, Laronga C, Harbrecht BG, Jensen EH, Fry DE, et al. American College of Surgeons and Surgical Infection Society: Surgical Site Infection Guidelines, 2016 Update. Journal of the American College of Surgeons. 1 de enero de 2017;224(1):59-74. Disponible en: 10.1016/j. jamcollsurg.2016.10.029

4. National Healthcare Safety Network, Centers for Disease Control and Prevention. Surgical site infection (SSI)event. January 2017. Accessed January 25, 2017. Disponible en: https://www.cdc.gov/nhsn/PDFs/ pscManual/9pscSSIcurrent.pdf

5. Bonifacio M, María S. Malnutrición como factor de riesgo asociado a las infecciones de sitio operatorio del departamento de Cirugía general del Hospital Nacional Daniel Alcides Carrión en el periodo enero-junio 2017. Universidad Ricardo Palma [Internet]. 2018 [citado 11 de septiembre de 2019]; Disponible en: http://repositorio.urp.edu.pe/ handle/URP/1178

6. López Plaza B, Bermejo López LM. Nutrición y trastornos del sistema inmune. Nutrición Hospitalaria. 2017;34:68-71. Disponible en: http:// dx.doi.org/10.20960/nh.1575.

7. Tarqui-Mamani C, Sanchez-Abanto J, Alvarez-Dongo D, EspinozaOriundo $\mathrm{P}$, Jordan-Lechuga T. Prevalencia de anemia y factores asociados en adultos mayores peruanos. Rev Peru Med Exp Salud Publica. diciembre de 2015;32:687-92. Disponible en: https://www. scielosp.org/scielo.php?pid=S1726-46342015000400009\&script=sci_ arttext\&tlng=en
8. Stauder R, Valent $P$, Theurl I. Anemia at older age: etiologies, clinical implications, and management. Blood. 1 de febrero de 2018;131(5):50514. Disponible en: https://doi.org/10.1182/blood-2017-07-746446

9. Pires Corona L, Drumond Andrade FC, de Oliveira Duarte YA, Lebrao $\mathrm{ML}$. The relationship between anemia, hemoglobin concentration and frailty in Brazilian older adults. J Nutr Health Aging. 1 de noviembre de 2015;19(9):935-40. Disponible en: https://doi.org/10.1007/s12603-0150502-3

10. Sattar F, Sattar Z, Zaman M, Akbar S. Frequency of Post-operative Surgical Site Infections in a Tertiary Care Hospital in Abbottabad, Pakistan. Cureus. 2019:11(3). DISPONIBLE EN: 10.7759/cureus.4243

11. Marin A, Rooseveit E. El hemograma completo como parametro predictor de riesgo de infección en sitio operatorio, en los pacientes operados en el servicio de cirugia del HRDC en el año 2017. Universidad Nacional de Cajamarca. 2019. Disponible en: http://repositorio.unc.edu. pe/handle/UNC/2963

12. Molina Soria JB, Lobo Támer G, Pérez de la Cruz AJ, Ruiz-López MD. Prevalencia de desnutrición al ingreso en un hospital general básico. Nutr Hosp 2017;34:1390-1398. Disponible en: http://dx.doi. org/10.20960/nh.1133

13. Bacardí Zapata PA, Páez Candelaria Y, Jones Romero O, Romero García LI, Ricardo Ramírez JM, Gondres Legró KM. Hipoalbuminemia e infección postoperatoria en una unidad de atención al grave. Cuba y Salud. 2017;12(3):24-9.n Disponible en : https://www.medigraphic. com/pdfs/cubaysalud/pcs-2017/pcs173e.pdf

14. Salvetti DJ, Tempel ZJ, Gandhoke GS, Parry PV, Grandhi RM, Kanter $A S$, et al. Preoperative prealbumin level as a risk factor for surgical site infection following elective spine surgery. Surg Neurol Int. 8 de octubre de 2015;6(Suppl 19):S500-3. DISPONIBLE en: 10.4103/21527806.166893 